\title{
Timing the shift in retinal local signs that accompanies a saccadic eye movement
}

\author{
J. SCOTT JORDAN and WAYNE A. HERSHBERGER \\ Northern Illinois University, De Kalb, Illinois
}

\begin{abstract}
The phantom array was used to probe the time course of the shift in retinal local signs that accompanies a saccadic eye movement. The phantom array materializes when one saccades in the dark across a point light source blinking 120 times per second. One sees a stationary array of flashes-the first materializes discretely near the intended endpoint of the saccade, and subsequent flashes materialize progressively closer to the actual position of the blinking light. Four trained observers indicated the perceived location, relative to the phantom array, of a 1-msec marker flash (M) produced by two LEDs (light-emitting diodes) that vertically bracketed the blinking light. The marker was seen as spatially coincident with the first flash when it flashed 80 to 0 msec before the saccade, and was seen as spatially coincident with either the first flash or the actual position of the blinking light when it flashed more than $80 \mathrm{msec}$ before the saccade, indicating, respectively, that the shift is presaccadic and rather abrupt.
\end{abstract}

The perceived location of a stationary object remains relatively constant across saccadic eye movements, despite the fact that the retinal locus of the object's image does not. This phenomenon is commonly referred to as visual direction constancy (Shebilske, 1977). Theorists claim that the nervous system accomplishes this perceived constancy across saccadic shifts in eye position by producing a similar shift in the spatiotopic coordinates (local signs) of the retina via a neural signal representing eye position (Bridgeman, 1986; Grüsser, 1986; Hallett \& Lightstone, 1976a, 1976b; Hansen \& Skavenski, 1985; Hershberger \& Jordan, 1992; Honda, 1989; Matin, 1972, 1982; Shebilske, 1976; Skavenski, 1972; Steinbach, 1987). Because the exact nature of this neural signal is unknown, it is commonly referred to as, simply, the extraretinal signal. What is known is that the shift in retinal local signs brought about by this putative extraretinal signal is not synchronized with the shift in eye position. This is evidenced by the fact that the location of a brief $(1-\mathrm{msec})$ flash of light presented in the dark at some point during a saccade, including the saccadic latency, is reliably misperceived (see, e.g., Matin, 1972; O'Regan, 1984).

Hershberger (1987) recently reported an illusion of visual direction that he refers to as the phantom array, which can be used to systematically measure the time

This paper is based upon a doctoral dissertation by J.S.J. at Northern Illinois University. We gratefully acknowledge the assistance of Mike Anderson, Chris Dalmares, Erin Powell, and Katherine Van Winkle in the data collection and thank W. T. Powers for his assistance in the computer programming. We also wish to express our appreciation to W. Becker for his comments on an earlier version of this article. Requests for reprints should be sent to J. S. Jordan, Department of Psychology, Saint Xavier University, 3700 West 103rd Street, Chicago, IL 60655 .

-Accepted by previous editor, Charles W. Eriksen course of such perisaccadic misperceptions relative to the saccade, thus allowing one to quantify the asynchrony between the saccadic and extraretinal shifts. Specifically, when one saccades in the dark across a point light source blinking at approximately $120 \mathrm{~Hz}$, the blinks painted upon the moving retina produce an array of dot-like afterimages that materialize sequentially in the direction opposite the saccade. The fact that one sees an array indicates that the saccadic and extraretinal shifts are asynchronous, because if they were synchronous, the retinal local signs would be shifted in the direction of the saccade at the same rate as the eye, and every flash would appear in the same place (i.e., the true location of the flashing light).

Given that the array is a product of the saccadic extraretinal asynchrony, the temporal and spatial aspects of the array itself can be used to investigate the temporal and spatial nature of the asynchrony. For example, suppose that the first dots to materialize in the array appear to move in the direction of the saccade as the later dots materialize. This would indicate that the retinal local signs are shifting gradually, in the direction of the saccade, during the saccade. On the other hand, suppose that the first dots in the array do not appear to move while the later dots materialize, but rather appear to remain in the same spatial location throughout the saccade. This would indicate that the retinal local signs are not changing during the saccade and, thus, must change either before (i.e., during the saccadic latency) or after the saccade.

Hershberger and Jordan (1994) report that subjects reliably perceive the phenomenon in the latter fashion. That is, subjects claim that once a dot in the array materializes, its perceived spatial location remains constant as the remaining flashes materialize, indicating that the shift is completed either before or after the saccade. Subjects further claim that the entire array appears on the side of the 
blinking light that is associated with the saccadic target. For example, during a rightward saccade, the first dot in the array discretely materializes somewhere near the intended endpoint of the saccade, and the subsequent dots in the array materialize at points farther and farther to the left until, upon the completion of the saccade, the last dot materializes near the actual location of the blinking light. This means that the first flash in the array appears near the intended endpoint of the saccade before the saccade begins. Thus, the shift in retinal local signs must be presaccadic.

The moment at which the first dot in the array materializes indicates the moment, relative to the saccade, at which the extraretinal signal produces a shift in the retinal local signs. The purpose of the present study was to determine this moment.

\section{METHOD}

\section{Observers}

Four undergraduate students at Northern Illinois University, 2 males and 2 females, were recruited to participate in the present study as psychophysical observers. All 4 were between the ages of 21 and 26, had normal, uncorrected vision, and had no prior experience with eye-movement research. Each observer earned course credit for participating in the experiment.

\section{Apparatus}

Visual displays were generated by means of an array of 11 lightemitting diodes (LEDs) mounted on a black, frontoparallel screen situated $160 \mathrm{~cm}$ from the seated observer's eyes (see Figure 1). Each LED ( $5 \mathrm{~mm}$ in diameter) subtended a visual angle of $.18^{\circ}$. LEDs $1,2,3,4$, and 5 were positioned in a horizontal row at eye level, with LED 4 located directly in front of the observer's right eye. LEDs 1 and 5 were located $5^{\circ}$ to the left and right of LED 4, respectively. LEDs 2 and 3 were located $3.75^{\circ}$ and $2.5^{\circ}$ to the left of LED 4 , respectively. LEDs 2,3 , and 4 were each vertically bracketed by two equally spaced LEDs $\left(.38^{\circ}\right.$ on center).

The displays generated by these LEDs were viewed in an otherwise darkened room, and an observer registered his/her judgments about a display by pressing buttons on a response panel. The buttons (B1, B2, B3, B4, B5, and B6) were situated in such a way that when the observer placed his/her right hand flat upon the response panel, B1 was located under the thumb, and B2 through B5 were each situated under the tip of one finger. The sixth button (B6) was located in the upper left corner of the response panel and

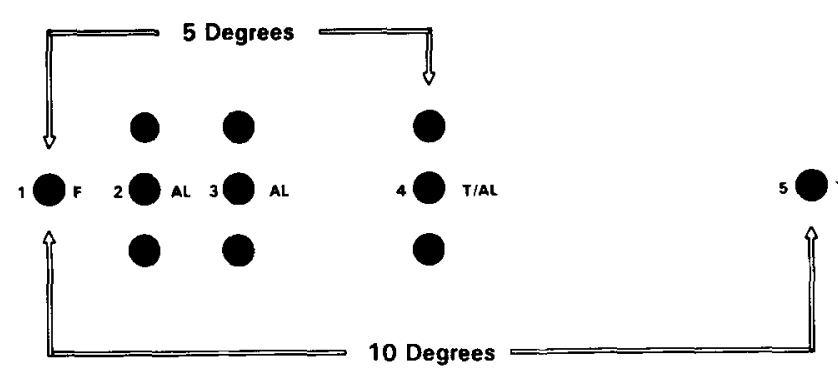

Figure 1. Arrangement of LEDs used to generate visual displays. LEDs 1-5 are numbered on the left. The letters to the right of LEDs 1-5 indicate the function of that LED. $F=$ fixation light, $A L=$ array light, $\mathbf{T}=$ target light.

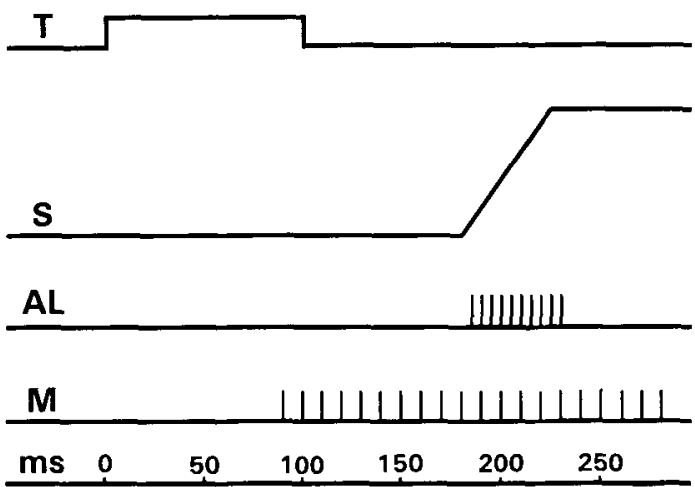

Figure 2. The chronology of events comprising the stimuluspresentation part of each trial, $F=$ fixation light, $T=$ target light, $\mathbf{S}=$ saccade from $\mathrm{F}$ to $\mathrm{T}, \mathrm{AL}=$ array light, $\mathbf{M}=$ marker flash; the bottom trace indicates milliseconds in relation to $T$ onset.

was operated by the thumb of the observer's left hand. The response panel and the LEDs were wired to a computer located in an adjacent room where the experimenter controlled the experiment.

A Gulf + Western Eye-Trac Model 200 was used to monitor the horizontal movements of the observer's right eye. The Eye-Trac is an infrared system, accurate to within $15^{\prime}$ of arc; its response time is $4 \mathrm{msec}$. The Eye-Trac signal was fed both to a storage oscilloscope and to the computer. The storage oscilloscope allowed the experimenter to observe the nature of the eye movement as it occurred, and the computer allowed the experimenter to digitize and store the eye movement as well as display it later on the computer's monitor.

\section{Procedure}

Trial configuration. Each trial comprised three parts: (1) an initial period during which the Eye-Trac was calibrated, (2) a stimuluspresentation period during which several LEDs flashed and the observer saccaded, thus experiencing the phantom array, and (3) a final judgment period during which the observer indicated his/her judgments about the phantom array by pressing buttons on the response panel.

Calibration. Although head-movement artifacts were kept to a minimum by using a bite board with dental wax, the apparatus was calibrated prior to each trial. To do this, the observer carefully fixated LED 1 and, while doing so, pressed a button on the response panel. This triggered the Eye-Trac to sample eye position at $1000 \mathrm{~Hz}$ (i.e., once each millisecond) for $100 \mathrm{msec}$. The observer then carefully fixated LED 5 and, while fixating the LED, pressed a button on the response panel, triggering the Eye-Trac to sample eye position at $1000 \mathrm{~Hz}$ for $100 \mathrm{msec}$. The horizontal distance between LEDs 1 and 5 subtended a visual angle of $10^{\circ}$; thus, the Eye-Trac voltages could be converted to degrees of visual angle. This made it possible to measure the size of the saccades and to ensure that the LED flashes were striking the appropriate groups of retinal loci.

Stimulus presentation. Figure 2 illustrates the chronology of events comprising the stimulus-presentation part of each trial. For each trial, two LEDs defined the size of the saccade. LED 1 produced a red point of fixation (F) for a randomly varied period of time ranging from 1,000 to 2,000 msec (the unpredictable duration of $F$ reduced the frequency of anticipatory saccades). Exactly 50 msec after the offset of F, either LED 4 (located $5^{\circ}$ to the right of F) or LED 5 (located $10^{\circ}$ to the right of F) produced a brief, 
100 -msec red flash that served as a saccadic target $(\mathrm{T})$. The observer, having been told to "follow the red light," saccaded (S) from $\mathrm{F}$ to T. A 150 - to 250 -msec latency normally exists between target onset and the actual initiation of a saccadic eye movement (Robinson, 1975); consequently, both $\mathrm{F}$ and $\mathrm{T}$ were extinguished before the eyes began to move.

Immediately upon the offset of $F$, the computer began to sample eye position at a rate of $1000 \mathrm{~Hz}$. Also, each millisecond, the computer compared the current sample with the prior one. (An increase in Eye-Trac scores indicated rightward eye movement, whereas a decrease in Eye-Trac scores indicated leftward eye movement.) A series of five consecutive increases in the values of the Eye-Trac samples was taken to indicate that the saccade from $F$ to $T$ had begun $5 \mathrm{msec}$ before; this very simple and reliable algorithm ensured that any error in identifying the beginning of a saccade was $<5 \mathrm{msec}$, maximum, and near 0 on the average. When this condition was met, one $\operatorname{LED}(2,3$, or 4$)$ began to flash green at a rate of $200 \mathrm{~Hz}$ (i.e., for $1 \mathrm{msec}$ out of every 5). The computer then determined, during every millisecond iteration, whether or not the eye had yet completed the saccade. The saccade was defined as complete when the value of the current Eye-Trac sample was either equal to or less than the value of the immediately previous sample. When this condition was met, the green LED stopped flashing. As the observer saccaded between $F$ and $T$, the green flashing LED served to generate the phantom array and is thus referred to as the array light (AL). The temporal relationship between the onset and completion of the saccade and the onset and offset of the array light is illustrated in Figure 2.

At a predetermined moment following the onset of $T$, both of the yellow LEDs bracketing the blinking array light produced a brief, 1 -msec yellow flash. The observer was to judge the point in the green phantom array that was coincident with the yellow flashes. Thus, the two yellow LEDs served as a marker (M). The possible moments during the trial when a marker could flash are depicted in Figure 2. How these moments were determined will be discussed later.

The luminance of the red, green, and yellow LEDs serving as target, array light, and marker were 175,125 , and $80 \mathrm{~mL}$, respectively. Also, LED 4 was a bipolar LED; thus, it could flash either red or green depending upon the criteria for a particular trial.

Judgments. At the end of the stimulus presentation, the observer used the buttons on the response panel to report observations of two types.

The first observation was whether or not the observer had seen the phantom array on that trial. B1 was pressed to indicate yes and B2 was pressed to indicate no. If an array had been seen, the observer reported a second type of observation.

The second observation indicated where the marker appeared relative to the phantom array painted by the blinking array light; this is called the marker-position judgment (MPI). The observer pressed the leftmost button, B1 (under the thumb), to indicate that the marker had appeared at the leftmost end of the array, and the rightmost button, B5 (under the little finger), to indicate that it had appeared at the rightmost end of the array. Buttons B2, B3, and B4 were pressed to indicate the inner left, middle, and inner right aspects of the array, respectively. The observer pressed the sixth button (B6, located in the upper left corner of the response panel) to indicate that he/she did not see a marker flash.

Because the apparent rightward displacement of the flashes comprising the phantom array reflect a shifting of retinal local signs, the observers' marker-position judgments reflect the moment when this shift occurs. That is, a marker flashed immediately after the shift has occurred (has been completed) and before the saccade has begun should appear to be located at the right end (beginning) of the array. Consequently, a marker flash that appears coincident with the rightmost end of the array (results in a response of B5) represents the perisaccadic moment when the shift in retinal local signs has just been completed. A marker flash that occurs before the shift in local signs or after the completed saccade should appear coincident with the left end of the array and result in a switch setting of B1. Marker flashes that occur while the eye is moving should appear to lie somewhere in the middle of the array and result in a switch setting of B2, B3, or B4.

It should be mentioned that the phrase "rightmost end of the array" included those trials during which the marker was seen a bit to the right of the right end of the array. This circumstance arose because the array light did not begin to flash until after the onset of the saccade. Thus, if the marker flashed before the onset of the saccade, the flash struck a retinal locus to the left of that struck by the first flash in the array and, thus, appeared at a point in space a bit to the right of the right end of the array. Likewise, the phrase "leftmost end of the array" included those trials during which the marker was seen a bit to the left of the left end of the array. This circumstance arose due to the oscillation rate of the array light (i.e., $200 \mathrm{~Hz}$, or one flash every $5 \mathrm{msec}$ ). Specifically, because the array light flashed at $200 \mathrm{~Hz}$, an array flash that occurred $4 \mathrm{msec}$ or less before the end of the saccade necessarily represented the last flash in the array. This caused a marker flash that occurred after such an array flash to strike a retinal locus a bit to the right of the last array flash and, thus, be seen at a point in space a bit to the left of the last array flash.

\section{Experimental Design}

The primary dependent variable was the marker-position judgment; the primary independent variable was the moment of the marker flash. As stated above, it was expected that the markerposition judgments would vary as a function of the moment of the marker flash and, thereby, reveal the moment of the shift in the retinal local signs. Further, three other independent variables were manipulated in order to assess their influence upon the moment of the shift. These variables were target distance, array-light position, and target-distance predictability.

The moment of the marker flash. Saccadic latencies, measured in milliseconds following target onset, were collected for each observer during an initial training period. The average of each observer's latencies was used to predict the moment during experimental trials (measured in milliseconds following target onset) when the average saccade would be most likely to occur for that observer; this parameter is called the predicted saccadic onset (PSO). It is well documented that saccadic latencies vary with saccade size (Bartz, 1962; Saslov, 1967; White \& Eason, 1962), so separate predicted saccadic onsets were used for $5^{\circ}$ and $10^{\circ}$ targets. These values are listed in Table 1 , along with the average saccadic onset observed

Table 1

Predicted Saccadic Onsets (PSOs), Obtained Saccadic Onsets (OSOs), and Standard Deviations, as a Function of Target Distance

\begin{tabular}{|c|c|c|c|c|}
\hline \multirow[b]{3}{*}{ Observer } & \multicolumn{4}{|c|}{ Practice Sessions } \\
\hline & \multicolumn{2}{|c|}{$5^{\circ}$ Target Distance } & \multicolumn{2}{|c|}{$10^{\circ}$ Target Distance } \\
\hline & PSO & $S D$ & PSO & $S D$ \\
\hline 1 & 200 & 56 & 130 & 34 \\
\hline 2 & 193 & 55 & 176 & 56 \\
\hline 3 & 148 & 44 & 146 & 55 \\
\hline \multirow[t]{4}{*}{4} & 150 & 26 & 140 & 22 \\
\hline & \multicolumn{4}{|c|}{ Experimental Sessions } \\
\hline & \multicolumn{2}{|c|}{$5^{\circ}$ Target Distance } & \multicolumn{2}{|c|}{$10^{\circ}$ Target Distance } \\
\hline & OSO & $S D$ & OSO & $S D$ \\
\hline 1 & 147 & 36 & 139 & 34 \\
\hline 2 & 255 & 52 & 225 & 45 \\
\hline 3 & 149 & 30 & 141 & 29 \\
\hline 4 & 158 & 18 & 155 & 19 \\
\hline
\end{tabular}

Note-All data are in milliseconds. 


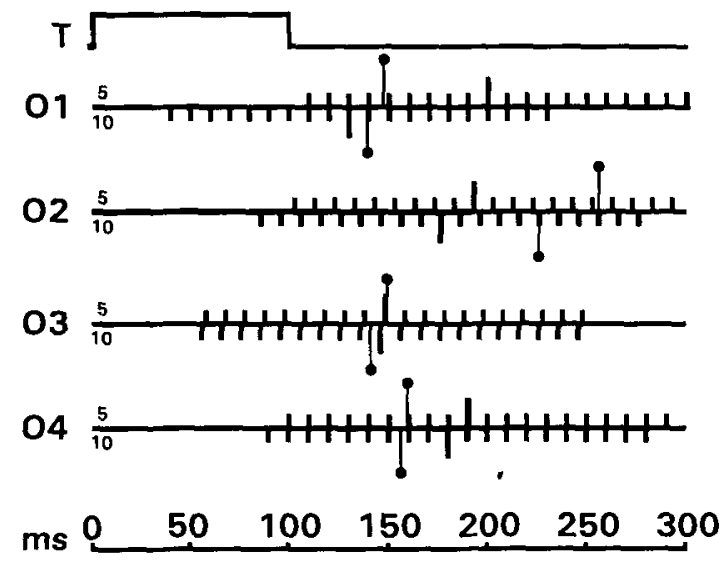

Figure 3. The average predicted saccadic onsets, the average obtained saccadic onsets, and the marker-onset windows for each observer, illustrated in terms of their temporal relationship to the saccadic target, T. O1, O2, 03, and $04=$ Observers $1,2,3$, and 4, respectively. The top of each observer's trace refers to the $5^{\circ}$ target distance; the bottom refers to the $10^{\circ}$ target distance. The large hash mark within each marker-onset window represents the predicted saccadic onset, and the crowned hash mark represents the obtained saccadic onset.

during the experimental sessions (i.e., observed saccadic onsets, or OSOs).

During the experimental sessions, the moment of the marker flash was systematically varied, across trials, in order to bracket the observer's predicted saccadic onset. Specifically, the presentations of the marker occurred within a 190-msec window that opened $90 \mathrm{msec}$ before and closed $100 \mathrm{msec}$ after the predicted saccadic onset. Within this marker-onset window, 20 unique marker-onset moments were utilized, each separated by $10 \mathrm{msec}$. (Figure 2 illustrates a marker-onset window properly situated around a hypothetical predicted saccadic onset of $180 \mathrm{msec}$.) The 190 -msec marker-onset window comprised three parts: a presaccadic portion lasting $90 \mathrm{msec}$, an intrasaccadic portion of about 40 to $50 \mathrm{msec}$ (a $10^{\circ}$ human saccade takes about $45 \mathrm{msec}$; see Robinson, 1968), and a postsaccadic portion of about $50 \mathrm{msec}$. Figure 3 illustrates the marker-onset windows used for the $5^{\circ}$ and $10^{\circ}$ targets for all the observers in relation to target $(\mathrm{T})$ onset. Observer 4 presented a special case, because his saccadic latencies were so brief. If the 190-msec-wide marker window had been centered on Observer 4's PSO, the early markers would have overlapped T, the saccadic target. Because the simultaneous occurrence of the two stimuli during practice trials masked the phantom array, the marker window was moved $40 \mathrm{msec}$ further into the time course of the saccade. That is, during testing, Observer 4's marker window was centered at PSO $+40 \mathrm{msec}$.

Variables potentially influencing the shift. As stated above, three independent variables other than the marker-onset moment were manipulated in the present study: (1) the distance between fixation and target, (2) the position of the array light, and (3) the predictability of the target distance.

Two target distances were used (i.e., the target was situated $5^{\circ}$ or $10^{\circ}$ to the right of fixation) to determine whether the moment of the shift in local signs varies as a function of saccade size. Arraylight position was varied (i.e., was either one fourth or one half of the distance between fixation and target) to ensure that any differences found between $5^{\circ}$ and $10^{\circ}$ saccades were attributable to target distance and not to the specific retinal receptors struck by the array light during the saccade. Target-distance predictability was manipulated in order to determine whether or not knowledge about the size of the impending saccade has an effect upon the moment of the shift in retinal local signs. These three variables were completely crossed with the 20 marker-onset moments, resulting in 160 unique trials.

Target distance was unpredictable during Trials 1-40 and 121-160. Within and across these two 40-trial blocks, target distance, arraylight position, and marker-onset moment were completely counterbalanced. Then, to make the target distance unpredictable, a random order of presentation was generated for the trials in each block.

Target distance was predictable during Trials 41-120. This was accomplished by presenting 20 trials of Target Distance A (either $5^{\circ}$ or $10^{\circ}$ ), followed by 40 trials of Target Distance B (i.e., the opposite of the target distance during the previous 20 trials), followed by 20 more trials of Target Distance $A$. Within each block of target distance, array-light position and marker-onset moment were completely counterbalanced. Target Distance $A$ was $5^{\circ}$ for the odd-numbered sessions, and $10^{\circ}$ for the even-numbered sessions. This manipulation ensured that constant errors due to order effects would be controlled for over the entire experiment.

Trial acceptability. Immediately following every trial, the saccade and its temporal relationship to the onset of the target, the onset of the array light, the offset of the array light, and the markeronset moment were displayed on the computer screen. The experimenter used this information to ensure that all events in the trial had occurred properly and that the flashes from the LEDs were striking the appropriate retinal loci (i.e., the retinal loci situated between the two retinal loci that had been measured during the calibration). Trials were repeated if (1) the Eye-Trac indicated that the observer did not complete the saccade correctly (i.e., the saccade anticipated the onset of the target, the observer blinked, etc.), (2) the observer indicated that some mishap occurred (i.e., he/she pressed the wrong button on the response panel, or he/she was not ready for the trial, etc.), or (3) the saccade was the wrong size by a factor of $20 \%$ or more. (Although the majority of his saccades fell within the $20 \%$ criterion range, Observer 4's criterion range was increased to $30 \%$ in order to minimize the number of discarded trials, because the accuracy of his saccades was highly variable.)

During trials with predictable target sizes (i.e., Trials 41-120), it was necessary to repeat all unsuccessful trials of Target Distance A before conducting any trials of Target Distance B so that target distance would remain predictable. In order to simplify matters, unsuccessful trials were repeated after every 20 th unique trial. Specifically, trials requiring repetition were rerun following Unique Trials $20,40,60,80,100,120,140$, and 160 .

Total number of trials and sessions. As stated above, each experimental session entailed the successful completion of all 160 trials. Each observer participated in 10 experimental sessions, resulting in 10 repetitions of each of the 160 unique trials for a total of 1,600 trials per observer. Observers 1,2 , and 3 participated twice a week for 5 weeks. Observer 4 completed his 10 sessions within a 2-week period.

\section{Training}

Each observer underwent an initial, three-phase training period. The first phase familiarized the observer with the task of saccading from the fixation point to the target (both $5^{\circ}$ and $10^{\circ}$ targets were utilized during all three phases of the training period). During such trials, the fixation point and the target were the only LEDs to flash. After about 20 such trials, the observer moved on to the second phase.

During the second phase, the observers were introduced to the phantom array. Thus, fixation and target flashed, as described above, and the array light flashed at $200 \mathrm{~Hz}$ during the saccade. After exposure to such trials, all 4 observers reported experiencing the phan- 
tom array. Specifically, they reported seeing a horizontal row of dots that appeared in its entirety on the right side of the array light; the first dot materialized near the intended endpoint of the saccade.

The third phase familiarized the observers with the marker. During these trials, the fixation point, the target, and the array light all flashed, just as they had in Phase 2 . In addition, the marker flashed for $1 \mathrm{msec}$ at a predetermined moment during each trial. The observers tended to describe the marker as a "hash mark" or a "column" that intersected the array in a perpendicular fashion. The experimenter then taught the observer how to use the control panel to indicate the position of the column within the phantom array.

The training period was ended when the observer indicated that he/she felt comfortable and confident in indicating all the required judgments via the response panel. The observer then participated in a final "dress rehearsal" session, in which all judgments were communicated via the response panel.

\section{RESULTS}

The first step in quantifying the time course of the marker-position judgments relative to the saccade was to determine, for every trial, the discrepancy in milliseconds between the onset of the marker flash and the onset of the saccade (i.e., the marker-saccade asynchrony, or MSA). This value was determined by subtracting the moment the saccade began from the moment the marker flashed, with both values measured from the target onset. A negative value indicates that the marker flashed before the onset of the saccade, and a positive value indicates that the marker flashed after the onset of the saccade. Trials were then sorted, separately for each subject, into 10 -msec bins (i.e., the bin who's midpoint equaled five encompassed marker-saccade asynchronies of 0-10 msec), according to the magnitude of the marker-saccade asynchrony. Then, for each observer, an average markerposition judgment was calculated for each bin containing at least 10 data points (trials). The results are plotted in Figure 4.

One can see in Figure 4 that the time course of the marker-position judgments (MPJ) fits the predicted pat-

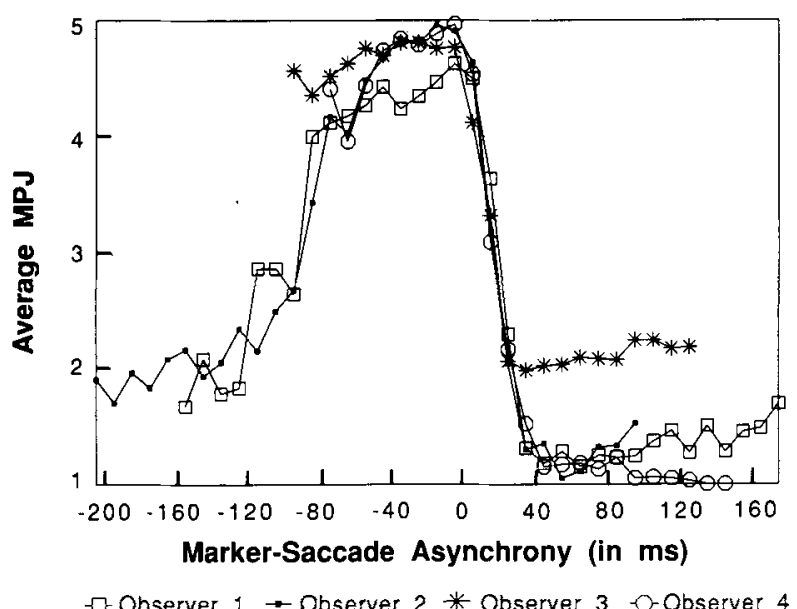

Figure 4. The observers' average marker-position judgments (MPJs) plotted as a function of the marker-saccade asynchrony.
Table 2

Average Saccadic Duration (in Milliseconds) and Standard Deviations, as a Function of Target Distance

\begin{tabular}{cccccc} 
& \multicolumn{2}{c}{$5^{\circ}$ Target Distance } & & \multicolumn{2}{c}{$10^{\circ}$ Target Distance } \\
\cline { 2 - 5 } \cline { 4 - 5 } Observer & Duration & $S D$ & & Duration & $S D$ \\
\hline 1 & 41 & 5 & 40 & 4 \\
2 & 35 & 4 & 48 & 7 \\
3 & 32 & 3 & 43 & 3 \\
4 & 30 & 3 & 43 & 4 \\
\hline
\end{tabular}

tern of results. Specifically, markers flashed at the beginning of the saccade (i.e., marker-saccade asynchrony equal to $0 \mathrm{msec}$ ) were seen at the right end of the array, markers flashed after the completion of the saccade (i.e., marker-saccade asynchronies equal to or greater than $40 \mathrm{msec}$ ) were seen at the left end of the array, and markers flashed later and later during the saccade were seen farther and farther to the left in the array. (Table 2 lists the average saccadic duration for each observer as a function of target distance.) Observers 1 and 2 have data in extremely negative bins (i.e., bin values from -80 to $-200 \mathrm{msec}$ ) because, as can be seen in Figure 3, Observer 1's saccades to the $10^{\circ}$ target and Observer 2's saccades to both targets tended to occur later than predicted, thus causing more markers to flash earlier than predicted in relation to the saccadic onset.

As stated previously, a marker-position judgment of 5 indicates a perisaccadic moment, by which time the shift in retinal local signs has taken place. One can see in Figure 4 that this telltale flash materializes well before the onset of the saccade. Specifically, markers that are flashed sometime between 80 and $0 \mathrm{msec}$ prior to the onset of the saccade are seen at the right end of the array. This indicates that the retinal local signs shift in the direction of the impending saccade somewhere around $80 \mathrm{msec}$ prior to the onset of the impending saccade (i.e., to the right in the present case). This does not necessarily indicate, however, that the shift is tied to the onset of the saccade, because the moment of the marker flash can be measured in two ways-in relation to the onset of the saccade (the marker-saccade asynchrony), or in relation to the onset of the target (the marker-target asynchrony).

To determine the event (i.e., the onset of the target or the onset of the saccade) to which the shift in retinal local signs is tied, each observer's data were sorted two ways. The first, the asynchrony between the marker and the saccade, has already been described. The second, the asynchrony between the marker and the target, was an experimental parameter. Specifically, the number of milliseconds intervening between the onset of the target and the moment of the marker flash was a fixed, independent variable. The number of marker-target asynchronies ( 40 , comprising 20 marker-onset moments for both the $5^{\circ}$ and $10^{\circ}$ target distances) and the number of trials at each markertarget asynchrony (40) was equal for all observers.

After both sorts were completed, the variance of the marker-position judgments at each marker-target asynchrony and within each marker-saccade asynchrony bin 
Table 3

Means of the Within-Bin Variance of the Marker-Position Judgments When Trials Were Sorted Into Bins According to Marker-Saccade Asynchronies and Marker-Target Asynchronies

\begin{tabular}{ccccc}
\hline & \multicolumn{2}{c}{ Asynchrony Classification Method } \\
\cline { 2 - 4 } Observer & \multicolumn{2}{c}{ Marker-Saccade } & & \multicolumn{2}{c}{ Marker-Target } \\
\cline { 2 - 5 } \cline { 4 - 5 } & Bins & $M$ & Bins & $M$ \\
\hline 2 & 38 & .87 & 40 & 1.30 \\
3 & 42 & .64 & 40 & 1.93 \\
4 & 32 & .25 & 40 & 1.93 \\
\hline
\end{tabular}

was determined so that the average bin variance from both sorting methods could be compared. Table 3 lists the average variance of the marker-position judgments by the sorting method for each observer. One can see in Table 3 that, in all cases, the variance of the marker-position judgments is smaller (i.e., the marker-position judgments are more consistent) when the moment of the marker flash is measured relative to the onset of the saccade. A $t$ test for each observer, which compared the average variance derived from each sorting method, confirmed this finding [Observer $1, t(76)=-2.58, p<.01 ;$ Observer $2, t(80)=$ $-7.99, p<.0001$; Observer $3, t(70)=-10.83, p<$ .0001 ; Observer $4, t(64)=-6.69, p<.0001]$.

Having determined that the shift in retinal local signs is tied to the onset of the saccade and not the onset of the target, the next step was to determine whether or not target distance, array-light position, target predictability, or session (first five vs. last five) influenced the moment of the shift. The plan was to conduct a multivariate analysis of variance. To make the results of such an analysis as easy to interpret as possible, the trials were divided into two groups that were analyzed separately: (1) trials in which the marker preceded the onset of the saccade (negative bins), and (2) trials in which the marker followed the onset of the saccade (positive bins). These analyses did reveal a few statistically significant variations in the relationship between marker-position judgments and the marker-saccade asynchronies; however, not one of the variables had the same effect across observers. This implies that these variables produced reliable, observerdependent idiosyncrasies rather than replicable alterations in the time course of the marker-position judgments.

The major results then, summarized in Figure 4, indicate that the shift in retinal local signs accompanying a saccadic eye movement is tied to the onset of the saccade and is typically completed about $80 \mathrm{msec}$ before the onset of the saccade. This presaccadic shift in retinal local signs is even more abrupt than a cursory inspection of Figure 4 suggests. At first glance, the gradual increase in the average marker-position value observed during the saccadic latency (i.e., during the negative bins) might lead one to believe that the shift in retinal local signs is itself gradual, because until the eye begins to move, the marker flashes are striking the same retinal locus. This means that any systematic changes in the average values must be reflecting changes in retinal local signs, and, if the values change gradually, so might the local signs. However, this conclusion is contraindicated by the relative frequency with which each marker-position value (i.e., 1, 2, 3, 4, or 5) was chosen at each bin. Figure 5 shows, for each observer, both the average marker-position judgment (MPJ) at each bin (upper function in each of the four sets) and the relative frequency with which each value was chosen at each bin (lower function in each of the four sets). If the average value for a particular bin faithfully indicates the perceived location of markers flashed during that bin, then the majority of flashes occurring during that bin should be seen at that point in the array corresponding to the average; that is, the mean and the mode should coincide. For example, one can see in Figure 5, for each observer, that during the saccadic eye movement, the modal value for successive bins generally decreases from 5 toward 1 , as does the average. However, within the negative bins, this correspondence is generally lacking; instead, the marker tends to be seen (i.e., is seen most frequently) at one end of the array or the other. For instance, in Observer 1's set (upper left), the modal value is a nearleft-end value (i.e., 1 or 2 ) within extremely negative bins (i.e., bin values ranging from -200 to -80 ), and then immediately becomes a right-end value (i.e., 5) within the less extremely negative bins (i.e., bins ranging from -80 to 0 ). Similarly, in Observer 2's set (upper right), the modal value is a near-left-end value (i.e., 2) during extremely negative bins, and then almost immediately becomes a right-end value (i.e., 5) during less extremely negative bins. Observers 3 and 4 do not have data in the extremely negative bins (lower left and lower right sets, respectively), so their data are essentially mute on this matter; however, note that their modal value is 5 for virtually every bin up until the onset of the saccade.

Apparently, the gradual increase in the average value observed during the negative bins generally reflects a gradually increasing probability that an abrupt shift in retinal local signs (taking less than $10 \mathrm{msec}$ ) has occurred by that time (i.e., by that bin value). This is not to say that the shift in local signs is perfectly discrete. Indeed, it appears as though the marker flash may have sometimes intercepted an abrupt but continuous shift in local signs in midflight. For instance, it is remarkable that for Observer 2 the modal value is 3 (albeit just barely) at the $-85-\mathrm{msec}$ bin, whereas it is 2 before that time and 5 after that time. It is equally remarkable, however, that for $\mathrm{Ob}$ server 1 , a value of 3 is the least frequent choice at this point of transition between left-end, and right-end judgments (i.e., at the -85 - and -95 -msec bins). It is therefore difficult to interpret the relative frequency of a marker-position judgment of 3 during the negative bins; that is, it is tempting to interpret a 3 as an intermediate value in a transition from 1 to 5 , but it may only indicate noise or a compromise between two equally potent alternatives, 1 and 5 . In any event, the shift in retinal local signs is abrupt. It is so abrupt that its time course exceeds the resolution of the present experimental methodology. 

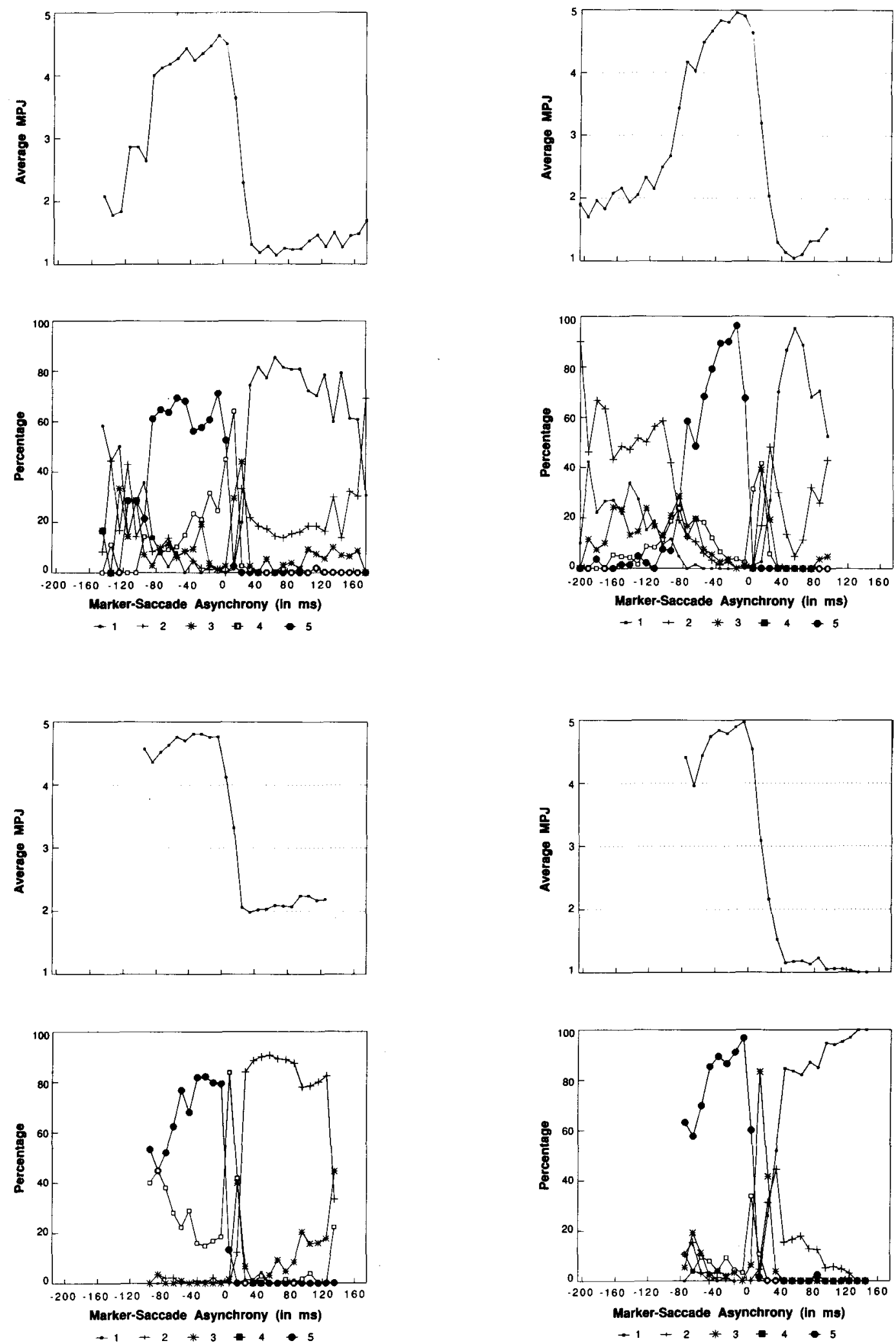

Figure 5. The average marker-position judgment (MPJ; top within each set) and the relative frequency of each MPJ (bottom within each set) shown for each marker-saccade asynchrony, for each observer. Observer $1=$ upper left set, Observer 2 = upper right set, Observer $3=$ lower left set, and Observer $4=$ lower right set. The bin width is $\mathbf{1 0}$ msec. Negative and positive bins represent trials in which the marker flashed before and after the saccade, respectively. 


\section{DISCUSSION}

The purpose of the present study was to time the shift in retinal local signs accompanying a saccadic eye movement. The present data indicate that this shift is rather abrupt (i.e., it requires less than $10 \mathrm{msec}$ ) and is complete roughly $80 \mathrm{msec}$ before the onset of the saccade.

The notion that the shift begins before the onset of the saccade is not new and has, in fact, been reported by many researchers (for a review, see Skavenski, 1990). This finding takes on new meaning however, when one considers Hershberger and Jordan's (1994) finding that once a flash in the phantom array materializes, its perceived location does not change as other flashes in the array materialize. This implies that the retinal local signs do not change during the saccade. Thus, the presaccadic shift found in the present study and cited in many others may represent the total shift that will occur. Further, both old and new data indicate that this presaccadic shift in retinal local signs may occur in a relatively discrete, quantum leap. For example, von Graefe (1854) reported that when a patient with a defective lateral rectus muscle attempted a saccade, the visual world appeared to jump in the direction of the intended eye movement, despite the fact that the eye did not move. This finding was later replicated in a more systematic fashion by Stevens et al. (1976), who found that subjects experiencing extraocular paralysis reported seeing the world "jump" in the direction of an attempted saccade. More recent support for the notion of a discrete, presaccadic quantum leap in the value of the retinal local signs can be found in the work of Grüsser, Krizic, and Weiss (1987), who had subjects report the perceived spatial location of a foveal afterimage while producing saccadic eye movements in the dark. Their subjects did not report seeing the afterimage move during the saccade; rather, they reported seeing the afterimage's postsaccadic position discretely displaced, from its presaccadic position, in the direction of the saccade.

All of the above findings, when considered together, indicate that the value of the retinal local signs, instead of sweeping through a range of values in an attempt to keep up with the moving eye, simply assumes two valuesthose appropriate for the pre- and postsaccadic direction of gaze.

Like Robinson $(1975,1981,1986)$, we assume that the oculomotor system monitors eye orientation by means of an efference copy and controls eye position by driving this efference copy into correspondence with a reference signal representing intended eye orientation. We suggest (Hershberger, 1987; Hershberger \& Jordan, 1992, 1994) that the local signs of the retina are determined by the intended rather than sensed orientation of the eye, and that retinal local signs shift abruptly when the neural signal representing intended eye orientation shifts discretely from one reference value to another.

Although we doubt that the neural signal representing sensed eye orientation (i.e., the inflow or outflow signal representing the oculomotor system's controlled variable) influences the retinas' local signs, our thesis does not imply that sensed eye orientation cannot influence judgments of egocentric direction (see Hershberger \& Jordan, 1992). The results of experiments utilizing coordinated movements such as hammer blows (Hansen \& Skavenski, 1985), refixation saccades (Hallett \& Lightstone, 1976a, 1976b), and manual pointing (Miller, 1993) as measures of perisaccadic target localization suggest that sensed eye orientation can sometimes influence judgments of egocentric direction. For example, Miller flashed a light onto his subjects' foveas as they made saccades between auditory targets presented in the dark and then had them point to the location of the flash. Using this behavioral method of assessing his subjects' ability to sense the changing orientation of their eyes during saccades (i.e., asking them to indicate their line of sight at the time of a perisaccadic flash), he found no presaccadic constant error of sensed eye orientation and estimated that almost immediately after the onset of a saccadic eye movement (e.g., $2 \mathrm{msec}$ ) the value of the signal representing sensed eye orientation begins a similar shift in the direction of the saccade. That is, he found that the signal for sensed eye position, whatever its source, whether inflow or outflow (Miller used Matin's neutral terminology, referring to the signal as an "extraretinal eye position signal"), is a relatively synchronous analog of eye orientation.

This relatively synchronous extraretinal eye position signal cannot, of course, account for the phantom array or any other form of that robust perisaccadic illusion of visual direction, which was first described by Matin and Pearce (1965) and subsequently replicated by a variety of experimental methods, including the measurement of refixation errors following perisaccadic target displacement (Dassonville, Schlag, \& Schlag-Rey, 1992; Hershberger \& Lucas, 1993; Honda, 1990). It follows, then, that the asynchrony of some other extraretinal eye position signal must be responsible for this robust illusionnamely, the oculomotor reference signal representing intended eye orientation.

It is sometimes assumed that the overall time course of the perisaccadic illusion of visual direction reflects the time course of the changing extraretinal eye position signal responsible for the illusion, but that assumption is unwarranted as well as contraindicated by the present data. If retinal local signs reflect the current value of an oculomotor reference signal representing intended eye orientation, any abrupt shift of intention will render the retinal local signs illusory, and the magnitude of the illusion will decrease gradually as the eye makes its way through the saccade.

It is also sometimes assumed that the gradual increase in the average magnitude of the constant error of visual direction observed during the $200 \mathrm{msec}$ just prior to a saccade reflects the rate of change of the extraretinal eye position signal responsible for the illusion, but this too is unwarranted (see Hershberger, 1987). The gradual increase may merely reflect a gradual increase in the probability that a perfectly discrete shift will have occurred 
by that time, much as we found to be the case in the present data. Consequently, the data that Dassonville et al. plotted in their (1992) Figure 3A, which is very similar to our present Figure 4, provide no basis for their claim that "the oculomotor system has access to only a damped representation of eye displacement" (p. 261). The oculomotor signal representing sensed eye displacement may be damped, but the oculomotor signal representing intended eye displacement is surely not.

The results of the present study are superficially similar, in several respects, to the findings of experiments requiring subjects to locate perisaccadic flashes of light relative to articulated visible backgrounds (Bischof \& Kramer, 1968; MacKay, 1970; O’Regan, 1984; Sperling \& Speelman, 1966). All these investigators have found flashes to be displaced in the direction of the saccade, and that this displacement is maximal at the beginning of the saccade and decreases at a rate that roughly parallels the saccade's. However, MacKay replicated Sperling and Speelman's demonstration that similar effects may also be produced by moving the visible background at saccadic velocities in the absence of a saccade.

In contrast to the methods of the experiments just cited, our subjects were not judging the position of the phantom array (nor any one of its parts) rela.ive to a visible background. Rather, they were judging the position of one element of the phantom array (the marker flash) relative to the remainder of the array. We know from personal observation (Hershberger, 1987) and prior research (Hershberger \& Jordan 1992, 1994) involving saccadic eye movements across a point light source, flashing in the dark as fast as $500 \mathrm{~Hz}$ (i.e., 1-msec flashes every other millisecond), that the first displaced flash in the phantom array is egocentrically displaced in the direction of the attendant saccade by an angular extent that is made visible by the array (i.e., the array subtends the angle). By including a distinctive element in the array, as we did (the marker came from the same azimuth location as all the other flashes in the array), we were able to determine that the first displaced flash occurs at least $80 \mathrm{msec}$ prior to the saccade.

Becker and Jürgens's (1975) finding that the amplitude of a saccade may be altered by retinal information that is presented as late as $80 \mathrm{msec}$ prior to the saccade implies that the oculomotor reference signal for the impending saccade is finalized just in time to generate the presaccadic portion of the perisaccadic illusion of visual direction.

Duhamel, Colby, and Goldberg (1992) recently reported finding neurons in the inferior parietal lobe of monkeys that shift their retinal receptive fields (local signs) to the same degree and in the same direction as an impending saccade well before the onset of the saccade (e.g., 80 msec or more).

The traditional interpretation of the perisaccadic illusion of visual direction is that the brain shifts the retinal local signs in order to compensate for an eye movement and generally dismisses the alternative possibility that the brain moves the eyes saccadically in order to compensate for an abrupt shift in retinal local signs. The present results obtained with the phantom array imply that the latter alternative is perhaps closer to the truth.

\section{REFERENCES}

BARTZ, A. (1962). Eye movement latency, duration, and response time as a function of angular displacement. Journal of Experimental Psychology, 64, 318-324.

BECKER, W., \& JüRGENS, R. (1975). Saccadic reactions to double-step stimuli: Evidence for model feedback and continuous information uptake. In G. Lennerstrand, P. Bach-y-Rita, C. C. Collins, A. Jampolsky, \& A. B. Scott (Eds.), Basic mechanisms of ocular motility and their clinical implications (pp. 519-524). New York: Pergamon.

BISCHOF, N., \& KRAMER, B. (1968). Untersuchungen und Überlegungen zur Richtungswahmehmung bei willkürlichen sakkadischen Augenbewegungen. Psychologische Forschung, 32, 195-218.

BRIDGEMAN, B. (1986). Multiple sources of outflow in processing spatial information. Acta Psychologica, 63, 35-48.

Dassonville, P., Schlag, J., \& Schlag-Rey, M. (1992). Oculomotor localization relies on a damped representation of saccadic eye displacement in human and nonhuman primates. Visual Neuroscience, 9, 261-269.

Duhamel, J.-R., Colby, C. L., \& Goldberg, M. E. (1992). The updating of the representation of visual space in parietal cortex by intended eye movements. Science, 225, 90-92.

GRÜSSER, O.-J. (1986). Interaction of efferent and afferent signals in visual perception: A history of ideas and experimental paradigms. Acta Psychologica, 63, 3-21.

GrüsSER, O.-J., KrIZIC, A., \& WeISs, L. (1987). Afterimage movement during saccades in the dark. Vision Research, 27, 215-226.

Hallett, P. E., \& Lighistone, A. D. (1976a). Saccadic eye movements to flashed targets. Vision Research, 26, 101-107.

Hallett, P. E., Lightstone, A. D. (1976b). Saccadic eye movements towards stimuli triggered by prior saccades. Vision Research, 16, 99-106.

HANSEN, R., \& SKavenSKi, A. A. (1985). Accuracy of spatial localizations near the time of saccadic eye movements. Vision Research, 25, 1077-1082.

Hershberger, W. [A.] (1987). Saccadic eye movements and the perception of visual direction. Perception \& Psychophysics, 41, 35-44.

Hershberger, W. A., Jordan, J. S. (1992). Visual direction constancy: Perceiving the visual direction of perisaccadic flashes. In E. Chekaluk (Ed.), The role of eye movements in perceptual processes (pp. 1-43). Amsterdam: Elsevier.

Hershberger, W. A., Jordan, J. S. (1994). The phantom array: A perisaccadic illusion of visual direction. Manuscript submitted for publication.

Hershberger, W. A., \& Lucas, D. R. (1993). Saccades to targets flashed during a prior saccade. Investigative Ophthalmology \& Visual Sciences, 34, 1498. (From Proceedings of the Annual Meeting of the Association for Research and Ophthalmology, Sarasota, FL, Abstract No. 3948)

Honda, H. (1989). Perceptual localization of visual stimuli flashed during saccades. Perception \& Psychophysics, 45, 162-174.

HoNDA, H. (1990). Eye movements to a visual stimulus flashed before, during, or after a saccade. In M. Jeannerod (Ed.), Attention and performance XIII: Motor representation and control (pp. 567-582). Hillsdale, NJ: Erlbaum.

MACKAY, D. M. (1970). Mislocation of test flashes during saccadic image displacements. Nature, 227, 731-732.

MATIN, L. (1972). Eye movements and perceived visual direction. In D. Jameson \& L. Hurvich (Eds.), Handbook of sensory physiology (Vol. 7, pp. 331-380). Heidelberg: Springer.

Matin, L. (1982). Visual localization and eye movements. In W. A Wagenaar, A. H. Wertheim, \& H. W. Leibowitz (Eds.), Eye movements and psychological processes (pp. 182-225). New York: Erlbaum. 
Matin, L., \& Pearce, D. G. (1965). Visual perception of direction for stimuli flashed during voluntary saccadic eye movements. Science, 148, $1485-1488$.

Miller, J. M. (1993). Egocentric localization of a brief perisaccadic flash. Investigative Ophthalmology \& Visual Sciences, 34, 1138. (From Proceedings of the Annual Meeting of the Association for Research and Ophthalmology, Sarasota, FL, Abstract No. 2142)

O'RegaN, J. K. (1984). Retinal versus extraretinal influences in flash localization during saccadic eye movements in the presence of a visible background. Perception \& Psychophysics, 36, 1-14.

Robinson, D. A. (1968). Eye movement control in primates. Science, 184, $1219-1224$.

RoBINSON, D. A. (1975). Oculomotor control signals. In G. Lennerstrand \& P. Bach-y-Rita (Eds.), Basic mechanisms of ocular motility and their clinical implications (pp. 337-374). New York: Pergamon.

Robinson, D. A. (1981). The use of control systems analysis in the neurophysiology of eye movements. Annual Review of Neuroscience, 4, 463-503.

Robinson, D, A. (1986). The systems approach to the oculomotor system. Vision Research, 26, 91-99.

SASLOV, M. (1967). Latency for saccadic eye movement. Journal of the Optical Society of America, 57, 1030-1033.

SHEBILSKE, W. L. (1976). Extraretinal information in corrective saccades and inflow vs. outflow theories of visual direction constancy. Vision Research, 16, 621-628.

SHEBILSKE, W. L. (1977). Visuomotor coordination in visual direction and position constancies. In W. Epstein (Ed.), Stability and constancy in visual perception (pp. 23-69). New York: Wiley.

SkavensKi, A. A. (1972). Inflow as a source of extraretinal eye-position information. Vision Research, 12, 221-229.

SkavenSki, A. A. (1990). Eye movement and visual localization of objects in space. In E. Kowler (Ed.), Eye movements and their role in visual and cognitive processes (pp. 263-287). Amsterdam: Elsevier. Sperling, G., \& SpeElmaN, R. A. (1966). Visual spatial localization during object motion, apparent object motion and image motion produced by eye movements. Journal of the Optical Society of America, 55, 1576-1577.

SteINBACH, M. J. (1987). Proprioceptive knowledge of eye position. Vision Research, 10, 1737-1744.

Stevens, J. K., Emerson, R. C., Gerstein, G. L., Kallos, T., NeuFled, G. R., Nichols, C. W., \& Rosenquist, A. C. (1976). Paralysis of the awake human: Visual perceptions. Vision Research, 15, 93-98.

von Graefe, A. (1854). Beiträge zur Physiologie und Pathologie der schiefen Augenmuskeln. Von Graefe's Archiv für Opthalmologie, 1, 1-18.

WhITE, C., \& EAson, R. (1962). Latency and duration of eye movements in the horizontal plane. Joumal of the Optical Society of America, 52, 210-213.

(Manuscript received June 8, 1992;

revision accepted for publication November 16, 1993.) 